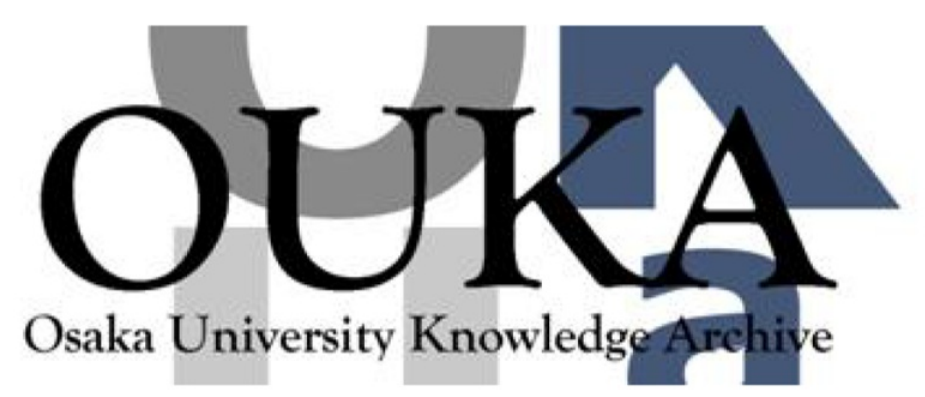

\begin{tabular}{|c|l|}
\hline Title & $\begin{array}{l}\text { I-V characteristics of single electron } \\
\text { tunneling from symmetric and asymmetric double- } \\
\text { barrier tunneling junctions }\end{array}$ \\
\hline Author(s) & Negishi, R; Hasegawa, T; Terabe, K et al. \\
\hline Citation & $\begin{array}{l}\text { APPLIED PHYSICS LETTERS. 90(22) p. 223112-1- } \\
\text { p. 223112-3 }\end{array}$ \\
\hline Issue Date & $2007-05-28$ \\
\hline oaire:version & VoR \\
\hline URL & https://hdl. handle. net/11094/3093 \\
\hline rights & \\
\hline Note & \\
\hline
\end{tabular}

Osaka University Knowledge Archive : OUKA

https://ir. Library. osaka-u. ac. jp/

Osaka University 


\title{
I- $V$ characteristics of single electron tunneling from symmetric and asymmetric double-barrier tunneling junctions
}

\author{
R. Negishi, ${ }^{\text {a) }}$ T. Hasegawa, K. Terabe, and M. Aono \\ Nanoscale Quantum Conductor Array Project, ICORP, JST, Kawaguchi, Saitama 332-0012, Japan \\ and Nano System Functionality Center, National Institute for Materials Science (NIMS), 1-1 Namiki, \\ Tsukuba, Ibaraki 305-0044, Japan \\ H. Tanaka and T. Ogawa \\ Research Center for Molecular-Scale Nanoscience, Institute for Molecular Science, 5-1 Higashiyama, \\ Myodaiji, Okazaki, Aichi 444-8787, Japan \\ H. Ozawa \\ Department of Electrical Engineering and Computer Science, Graduate School of Engineering, Nagoya \\ University, Furo-cho, Chikusa-ku, Nagoya 464-8603, Japan
}

(Received 15 April 2007; accepted 9 May 2007; published online 31 May 2007)

\begin{abstract}
$I-V$ characteristics of single electron tunneling from a symmetric and an asymmetric double-barrier tunneling junction (DBTJ) were examined. A single Au nanoparticle was trapped in nanogap whose size was precisely controlled using a combination of electron beam lithography and molecular ruler technique. Though the symmetric junction showed a monotonic rise with a bias beyond the Coulomb gap voltage, the asymmetric junction showed Coulomb staircases. The capacitance of the junction estimated from the fitting curves using the Coulomb conventional theory was consistent with the capacitance calculated from the observed structure. The authors quantitatively found the correlation between the electrical and structural properties of DBTJ. (C) 2007 American Institute of Physics. [DOI: 10.1063/1.2745252]
\end{abstract}

Coulomb blockade phenomena are of interest due to their potential application in single electron devices. The correlation between the structural and the electrical properties needs to be clarified so that the devices can be properly designed. The electrical characteristics of a single electron tunneling phenomenon are well described using a conventional theory ${ }^{1-3}$ on the basis of the double-barrier tunneling junction (DBTJ). The theory indicates that a highly asymmetric junction $\left(C_{1} R_{1} / C_{2} R_{2} \gg 1\right.$ or $\ll 1 ; C_{1}, C_{2}$ and $R_{1}, R_{2}$ represent the capacitance and the resistance in each junction) shows Coulomb staircases in the $I-V$ characteristics. When the tunneling rates of the two junctions are comparable $\left(C_{1} R_{1}\right.$ $\approx C_{2} R_{2}$ ), the Coulomb staircases vanish, and the $I$ - $V$ characteristics show a monotonic rise with a bias beyond the Coulomb gap voltage. Many experimental studies on DBTJ structures have been conducted using nanogap electrodes ${ }^{4,5}$ and a scanning tunneling microscope (STM) system. ${ }^{6-8} I-V$ curves with the Coulomb staircases and those with the monotonic rise were observed in these studies, which were explained based on the conventional theory. However, studies have not sufficiently provided a quantitative explanation that correlates the structural and the electrical properties of the DBTJ structures because of the high number of particles in the gap region ${ }^{4,5}$ and/or an uncertain gap size using STM. ${ }^{6-8}$ Here, we report $I-V$ curves measured with DBTJ structures having a single nanoparticle in a gap of a well-defined size, thereby enabling a quantitative explanation.

We have developed a method to fabricate a nanogap electrode device which has a well-defined gap size and a narrow width for containing a single nanoparticle in the gap region. ${ }^{13}$ The development involved combining selfassembled molecular (SAM) lithographic ${ }^{9-12}$ and electron

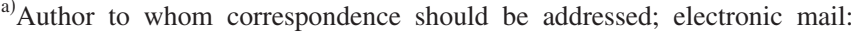
negishi.ryota@nims.go.jp
}

beam lithographic (EBL) techniques. SAM lithography enables precise control of the gap size in a unit of approximately $2 \mathrm{~nm}$ since the formation of a multilayer film composed of organic molecules and metal ions, which acts as a lift-off mask, can be controlled layer by layer (the thickness of the single SAM layer is approximately $2 \mathrm{~nm}$ ). EBL enables the design of gap electrodes, such as the position of the gap and the width of the electrodes, to be highly accurate. ${ }^{13}$ We demonstrated precise control by fabricating a nanogap with the desired size so that dodecanethiol-coated Au nanoparticles were placed in the nanogap electrodes, thereby enabling single electron tunneling phenomena to be observed. ${ }^{13}$ A scanning electron microscope (SEM) image of the device and the current-voltage $(I-V)$ characteristics clearly showed that the gap size matched the design.

In this study, we used our method to investigate the electrical and structural properties of symmetric and asymmetric DBTJ structures with a single Au nanoparticle.

Dodecanethiol-coated $\mathrm{Au}$ nanoparticles were prepared using the established method: ${ }^{14}$ (i) $3 \mathrm{ml}$ of a citrate-stabilized Au sol $\left(5 \times 10^{13}\right.$ particles $\left./ \mathrm{ml}\right)$ with an average particle size of $5 \mathrm{~nm}$ (British Biocell International Inc.) was mixed with $60 \mu \mathrm{l}$ of an approximately $20 \mathrm{mM}$ solution of $n$-dodecanethiol in ethanol for $30 \mathrm{~min}$; (ii) after ethanol $(18 \mathrm{ml})$ was added, the mixture was then centrifuged at $3500 \mathrm{rpm}$ for $1 \mathrm{~h}$; (iii) the precipitate was dried in air for $12 \mathrm{~h}$. The diameter of dodecanethiol-coated Au nanoparticle was estimated to be approximately $8 \mathrm{~nm}$ since the length of the dodecanethiol was about $1.5 \mathrm{~nm}$. The synthesized nanoparticles dissolved in chloroform were then dispersed on the nanogap devices by using spin casting at $1000 \mathrm{rpm}$ for $60 \mathrm{~s}$.

We prepared two different-sized gaps using four and five layers of the SAM films to make symmetric and asymmetric DBTJs with a single nanoparticle. The first and second metal electrodes composed of $\mathrm{Pt}(70 \mathrm{~nm}) / \mathrm{Ti}(10 \mathrm{~nm})$ and 

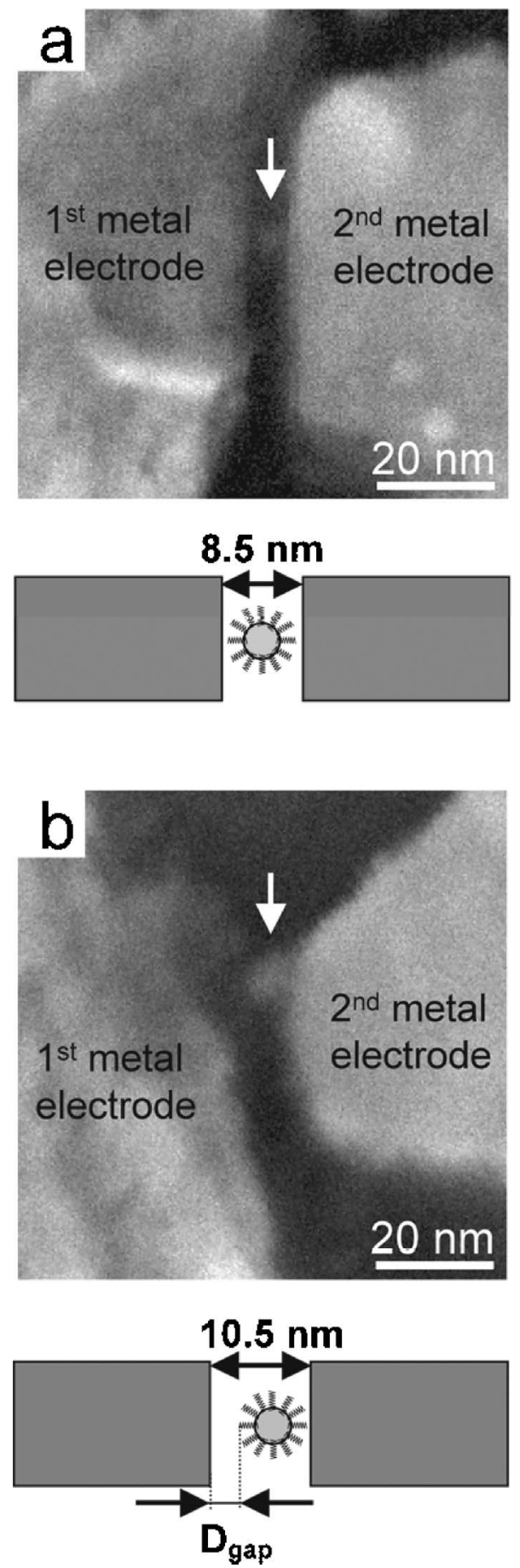

FIG. 1. SEM images of nanogap electrodes after the dispersion of Au nanoparticles. The arrows indicate the dispersed Au nanoparticles. The nanogap was (a) $8.5 \pm 0.5 \mathrm{~nm}$ and (b) $10.5 \pm 0.5 \mathrm{~nm}$. The nanogaps geometrically form a symmetric and an asymmetric junction. Illustrations of the gap regions are also shown in the lower part.

$\mathrm{Au}(15 \mathrm{~nm}) / \mathrm{Cr}(5 \mathrm{~nm})$ were formed on silicon oxide surface of a Si wafer by EBL patterning and metal deposition. After the width of electrodes was reduced to $50 \mathrm{~nm}$, most of the nanogaps could trap a single nanoparticle with the method.

Figures 1(a) and 1(b) show the SEM images of small $($ gap size $=8.5 \pm 0.5 \mathrm{~nm})$ and large $($ gap size $=10.5 \pm 0.5 \mathrm{~nm})$ nanogap devices after the dodecanethiol-coated Au nanoparticles were dispersed. It should be noticed that a single nanoparticle is trapped in each nanogap. Since the diameter of a Au nanoparticle, including the dodecanethiol, is approximately $8 \mathrm{~nm}$, the samples should work as a symmetric junc-
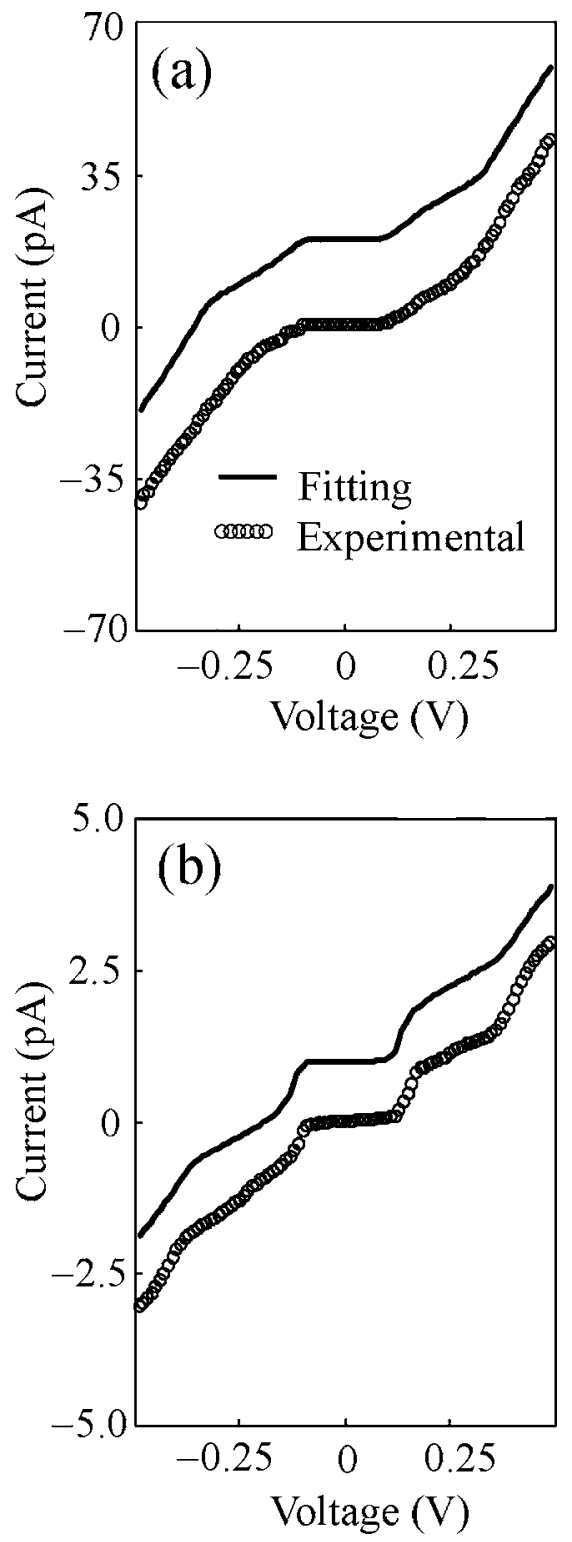

FIG. 2. I-V curves measured at $10 \mathrm{~K}$ (open circles) obtained from symmetric and asymmetric junctions shown in Figs. 1(a) and 1(b), respectively. The fitting curves (the solid lines) were obtained using the standard semiclassical formula (Refs. 1-3).

tion and an asymmetric junction bridged by a single nanoparticle. In fact, $\mathrm{Au}$ nanoparticles are in symmetric and asymmetric positions, as shown in the illustrations of the nanogap region in the lower part of Figs. 1(a) and 1(b).

Figures 2(a) and 2(b) show $I-V$ (I-V: open circles) curves measured at $10 \mathrm{~K}$ with the symmetric and asymmetric junctions shown in Figs. 1(a) and 1(b), respectively. The $I-V$ curves exhibited no current in the range of applied voltage from -0.1 to $0.1 \mathrm{~V}$. However, the $I-V$ curves measured at room temperature with the samples did not show a nocurrent region but showed a linear increase even in the zero bias region. This means that electron tunneling was suppressed because the charging energy of the nanoparticle was sufficiently larger than the thermal fluctuation energy $(\sim 0.86 \mathrm{meV})$ at $10 \mathrm{~K}$. At room temperature, the values of the two energies became closer, and the electron tunneling occurred even at a zero bias region. The results suggest that the Coulomb blockade phenomenon occurred at $10 \mathrm{~K}$ with a Coulomb gap voltage of $0.2 \mathrm{~V}$. 
TABLE I. Fitting parameters for theoretical Coulomb blockade curves as indicated by the solid line Figs. 2(a) and 2(b). $Q_{0}$ is the offset charges of a Au nanopartical.

\begin{tabular}{lcccccc}
\hline \hline & $\begin{array}{c}C_{1} \\
(\mathrm{aF})\end{array}$ & $\begin{array}{c}C_{2} \\
(\mathrm{aF})\end{array}$ & $\begin{array}{c}R_{1} \\
(\mathrm{G} \Omega)\end{array}$ & $\begin{array}{c}R_{2} \\
(\mathrm{G} \Omega)\end{array}$ & $Q_{0}$ & $C_{1} R_{1} / C_{2} R_{2}$ \\
\hline $\begin{array}{l}\text { Symmetric junction } \\
\text { Asymmetric junction }\end{array}$ & 0.72 & 0.76 & 3.9 & 3.7 & $0.001 e$ & $\approx 1.0$ \\
\hline \hline
\end{tabular}

One of the most revealing findings is the fact that the $I-V$ characteristics obtained from the symmetric and asymmetric junctions showed a monotonic rise and Coulomb staircases, respectively, with the bias beyond the Coulomb gap voltage, as shown in Figs. 2(a) and 2(b). This is consistent with the conventional theory based on the DBTJ structure. Though an extended method that accounts for the quantum effect for the single tunneling behavior has been reported, ${ }^{15}$ we analyzed the results using the conventional theory, which does not take into account the quantum effect because the influence of the quantum size effect on the electrical transport properties should be much smaller than that of the charging energy for a metal particle with diameter of $5 \mathrm{~nm} .{ }^{16}$

To determine the junction parameters, we used the standard semiclassical formula for a DBTJ structure ${ }^{1-3}$ to perform a curve fitting to the experimental data. The solid lines in Figs. 2(a) and 2(b) represent the fitting curves. The junction parameters used for the fitting are listed in Table I.

For the symmetric junction, two resistances (capacitances), $R_{1 s}\left(C_{1 s}\right)$ and $R_{2 s}\left(C_{2 s}\right)$, were almost the same and the time constants of the two junctions were comparable with each other $\left(R_{1 s} C_{1 s} \cong R_{2 s} C_{2 s}\right)$. We expected a vacuum region to be present in the junction because the gap size $(8.5 \pm 0.5 \mathrm{~nm})$ was slightly larger than the diameter of the dodecanethiol-coated Au nanoparticle $(8 \mathrm{~nm})$. The fitting result suggests that a vacuum gap was present in both junctions, and the sizes were almost the same.

For the asymmetric junction, the time constant ratio $R_{1 a} C_{1 a} / R_{2 a} C_{2 a}$ of the two junctions was estimated to be approximately 22 from the fitting results. This result means the electrical high asymmetry. We noticed that the parameters in the smaller junction of the asymmetric DBTJ, $R_{2 a}$ and $C_{2 a}$, were comparable with those in the symmetric DBTJ, suggesting that the vacuum gap there was almost the same size as the one in the symmetric DBTJ. The electrical dipole moment has been reported to be induced in a metallofullerene on the SAM layer. ${ }^{17}$ We think that the vacuum gap, which should be $0.25 \mathrm{~nm}$, is formed due to the Coulomb repulsive force between the surfaces of the SAM layer of the Au nanoparticle and the metal electrodes.

We calculated the capacitances of the junctions using the image charging method ${ }^{18}$ to check whether the fitting parameters are reasonable for the observed structural properties shown in Figs. 1(a) and 1(b). In the estimation, we assumed that a sphere with a diameter of $5 \mathrm{~nm}$ (Au nanoparticle) was covered by a high dielectric region $1.5 \mathrm{~nm}$ thick (the SAM layer of dodecanethiol), and the gap size was $8.5 \mathrm{~nm}$ $(10.5 \mathrm{~nm})$ for the smaller (larger) junction. We estimated the capacitance of the smaller junction with a vacuum gap of $0.25 \mathrm{~nm}$ to be $0.6-0.7 \mathrm{aF}$ using the dielectric constants of 3.0 for the SAM region ${ }^{19}$ and 3.9 for the $\mathrm{SiO}_{2}$ region. We evaluated the capacitance of the larger junction, which cor- responds to a junction between the first electrode and the nanoparticle in Fig. 1(b), to be $0.3-0.4$ aF by assuming the vacuum gap size of $2.25 \mathrm{~nm}$. The estimated values are consistent with the values obtained from the curve fitting. Thus, we believe that the conventional theory based on the DBTJ structure describes the single electron tunneling phenomena qualitatively and quantitatively.

In summary, we investigated the correlation between the structural and electrical properties of a DBTJ structure that was well defined using a combination of EBL and SAM lithographic techniques. ${ }^{13}$ With a bias beyond the Coulomb gap voltage, a symmetric structure shows a monotonic rise, and an asymmetric structure shows Coulomb staircases, as expected from the conventional DBTJ theory. In addition, junction parameters obtained from a fitting with the measured $I-V$ curves and the conventional DBTJ theory agree well with the calculated values using the structural properties of the formed DBTJ. We quantitatively found the correlation between the electrical and structural properties of the DBTJ by the electrical and geometrical characterization of the same junction structure. This fabrication method can be used for a single molecular nanodevice by using the functional switching molecules ${ }^{17,20}$ instead of the dodecanethiol.

A part of this work was supported by the Nanotechnology Support Project of the Ministry of Education, Culture, Sports, Science and Technology (MEXT), Japan and the Mitsubishi Foundation.

${ }^{1}$ D. V. Averin and K. K. Likharev, Mesoscopic Phenomena in Solids, edited by B. L. Altshuler, P. A. Lee, and R. A. Webb (Elsevier, Amsterdam, 1991), p. 173.

${ }^{2}$ M. Amman, R. Wilkins, E. Ben-Jacob, P. D. Maker, and R. C. Jaklevic, Phys. Rev. B 43, 1146 (1991).

${ }^{3}$ A. E. Hanna and M. Tinkham, Phys. Rev. B 44, 5919 (1991).

${ }^{4}$ D. L. Klein, R. Roth, A. K. L. Lim, A. P. Alivisatos, and P. L. McEuen, Nature (London) 389, 699 (1997).

${ }^{5}$ K. I. Bolotin, F. Kuemmeth, A. N. Pasupathy, and D. C. Ralph, Appl. Phys. Lett. 84, 3154 (2004).

${ }^{6}$ M. Dorogi, J. Gomez, R. Osifchin, R. P. Andres, and R. Reifenberger, Phys. Rev. B 52, 9071 (1995).

${ }^{7}$ K.-H. Park, J. S. Ha, W. S. Yun, M. Shin, K.-W. Park, and EL-Hang Lee, Appl. Phys. Lett. 71, 1469 (1997).

${ }^{8}$ H. Osman, J. Schmidt, K. Svensson, R. E. Palme, Y. Shigeta, and J. P. Wilcoxon, Chem. Phys. Lett. 330, 1 (2000).

${ }^{9}$ A. Hatzor and P. S. Weiss, Science 291, 1019 (2001).

${ }^{10}$ M. E. Anderson, R. K. Smith, Z. J. Donhauser, A. Hatzor, P. A. Lewis, L. P. Tan, M. W. Horn, and P. S. Weiss, J. Vac. Sci. Technol. B 20, 2739 (2002).

${ }^{11}$ M. E. Anderson, L. P. Tan, H. Tanaka, M. Mihok, H. Lee, M. W. Horn, and P. S. Weiss, J. Vac. Sci. Technol. B 21, 3116 (2003).

${ }^{12}$ H. Tanaka, M. E. Anderson, M. W. Horn, and P. S. Weiss, Jpn. J. Appl. Phys., Part 2 43, L950 (2004).

${ }^{13}$ R. Negishi, T. Hasegawa, K. Terabe, M. Aono, T. Ebihara, H. Tanaka, and T. Ogawa, Appl. Phys. Lett. 88, 223111 (2006).

${ }^{14}$ V. Santhanam, J. Liu, R. Agarwal, and R. P. Andres, Langmuir 19, 7881 (2003).

${ }^{15}$ O. Millo, D. Katz, Y. Cao, and U. Banin, Phys. Rev. Lett. 86, 5751 (2001).

${ }^{16}$ J. G. Hou, B. Wang, J. Yang, K. Wang, W. Lu, Z. Li, H. Wang, D. M. Chen, and Q. Zhu, Phys. Rev. Lett. 90, 246803 (2003).

${ }^{17}$ Y. Yasutake, Z. Shi, T. Ozaki, H. Shinohara, and Y. Majima, Nano Lett. 5, 1057 (2005).

${ }^{18}$ C. Gao, F. Duewer, and X. D. Xiang, Appl. Phys. Lett. 75, 3005 (1999).

${ }^{19}$ M. A. Rampi, O. J. A. Schueller, and G. M. Whitesides, Appl. Phys. Lett. 72, 1781 (1998).

${ }^{20}$ J. Chen, M. A. Reed, A. M. Rawlett, and J. M. Tour, Science 286, 1550 (1999). 